(C) 2015 IEEE. Personal use of this material is permitted. Permission from IEEE must be obtained for all other uses, in any current or future media, including reprinting/republishing this material for advertising or promotional purposes, creating new collective works, for resale or redistribution to servers or lists, or reuse of any copyrighted component of this work in other works.

http://dx.doi.org/10.1109/CAMAD.2015.7390521 


\section{Towards a Scalable Routing Approach for Mobile Ad-hoc Networks}

\author{
Tipu A.Ramrekha and Olayinka Adigun \\ UbiTech Ltd, GU2 7YG, London, United Kingdom \\ Email:\{Arvind, Olayinka\}@ubitechit.com
}

\author{
Alexandros Ladas, Nuwan Weerasinghe and Christos Politis \\ Wireless Multimedia \& Networking (WMN) Research Group \\ Faculty of Science, Engineering and Computing (SEC) \\ Kingston University London, KT1 2EE London, United Kingdom \\ Email: \{A.Ladas, Nuwan.Weerasinghe, C.Politis\}@kingston.ac.uk
}

\begin{abstract}
The Internet is evolving towards a two-fold architecture that will comprise of traditional infrastructure based networks as well as emerging self organised autonomic peripheral networks. Such Internet peripheral networks are being termed as the Internet of things (IoT) whereby smart objects and devices will be connected together in a fully distributed fashion to provide ubiquitous services through pervasive networking. Mobile Ad hoc Networks (MANETs) is regarded as one of the pervasive self-organised networks that will play a major role in autonomic future internet communication. There are several wellknown challenges to be addressed in order to enable MANET deployments of large islands of interconnected smart devices. Therefore, in this paper, we perform simulations using the ns-2 software, for various well-known routing protocols for MANETs in order to determine the scalability of these protocols. This paper analyses the scalability of the routing protocols with respect to routing overhead required by approaches while also considering the packet delivery latency, which is an important Quality of Service $(\mathrm{QoS})$ metric.
\end{abstract}

Keywords-MANETs, Scalable Routing, IoT

\section{INTRODUCTION}

Future Internet is embracing the Internet of Things (IoT) concept where smart devices should form fully distributed peripheral networks. The resulting architecture would consist of a two-fold paradigm of the current infrastructure based Internet platforms as well as a novel fully distributed peripheral network for IP based ubiquitous services. Such peripheral networks would then be interconnected to the Internet cloud using gateways or other backbone carriers. This will allow islands of autonomic smart devices to interchange data both with the Internet cloud and across remote machine to machine (M2M) communication based "islands of things". Generally, there are different boundaries that are used to differentiate among ad-hoc networking types namely single-hop or multihopped communication as well as static or mobile ad-hoc networking. Other boundaries that are also employed include data-centric or IP-centric routing paradigms or most recently, the nature and purpose of devices e.g. sensor devices. These paradigms and boundaries of distributed ad-hoc networks are described in Fig. 1. It can be hence foreseen that the future Internet paradigm is shifting from user operated devices that require "information push" services towards autonomous networks of smart devices delivering user-centric pervasive "information pull" services via M2M communication through the IoT.

The MANET flavour of self-organised peripheral future internet networks presents the highest level of challenges as described by Conti and Giordano [1]. The added research interest on routing for MANETs stems from the fact that multi-hop MANETs requires a new routing approach to route packet in a topology where any devices can assume any roles from a data source, destination or router. Currently the Internet Engineering Task Force (IETF) MANET Working Group (WG) are leading standardisation efforts towards a routing protocol for MANETs. Emanating from the 2 chartered tracks of MANET WG are the proactive Optimised Link State Routing (OLSR) and Ad-hoc On-demand Distance Vector (AODV) protocols deemed appropriate for standardisation. However, one of the main challenges to be addressed before the widespread deployment of MANETs remains the scalability of the routing approaches discussed at the IETF and in literature [1], [2], [3], [4]. Also, recent research in the field of scalable MANET routing protocols [5], [6], [7] indicate that while OLSR and AODV approaches, also termed as flat routing approaches, do offer a strong MANET routing basis, they do not provide an appropriate solution to the problem. This is mainly because proactive approaches have been found to be more appropriate for small sized networks while reactive protocols scale better for lager MANETs. Thus, in order to benefit from the strengths of each approach, several hybrid approaches including hybrid adaptive approaches are being proposed as a precursor towards enabling scalable MANET solutions for the future IoT.

Henceforth, in this paper, we focus on the various scalable approaches proposed both at the IETF MANET WG and that are well-known in literature. In this perspective, it is important to discuss the efficiency and effectiveness of these various adaptive approaches as recommended in RFC 2501 [2]. In particular, multimedia services are expected to be widely popular in these networks and thus the corresponding Quality of Service (QoS) requirements. As a result, we provide the performance evaluation for the presented scalable routing protocols considering the end-to-end message delivery latency and overhead performances in order to distinguish their suitability for the deployment in realistic IoT scenarios.

\section{TRADITIONAL OR FLAT NETWORKING APPROACHES}

MANETs require autonomic self-management because of its distributed nature as described in RFC 2501 [2] and in literature [1]. Essentially, MANET routing protocols need to have an autonomic mechanism for route discovery and route maintenance. This distributed detection of adjoining 


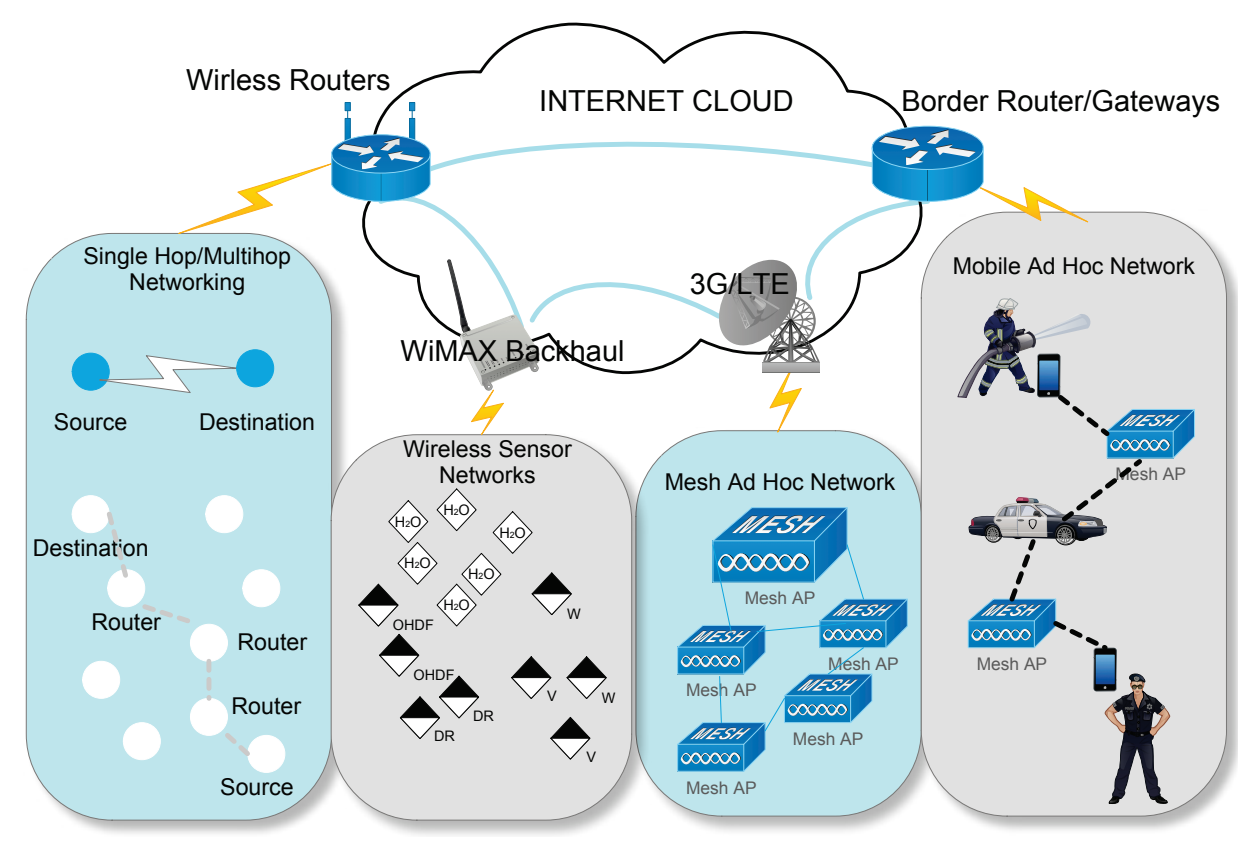

Fig. 1. Categorisation of future Internet Peripheral Networks

mobile nodes in obstacle prone wireless environments allows a completely self-managed decentralised computation of routes for end-to-end multi-hop communication. At the current stage, there are two main approaches that are being investigated by the IETF MANET WG in its proactive and reactive tracks for unicast routing although the WG also investigates the possibility of constrained flooding for multicasting. The protocols are specified in Internet-Drafts (I-Ds) or Request for Comments (RFCs) available at the IETF MANET WG Charter documents webpage. It is important to note that the basic challenges such as distributed tree construction, loop freedom, collision avoidance and packet formatting have been solved by the MANET WG protocols and are out of discussion scope within this article.

Firstly, the reactive AODV protocol [1] uses a Route Request (RREQ) broadcast packet to locate destinations when required on-demand by the data source. Although such an approach generates routing overhead on an on-demand basis only, it nevertheless requires added latency for route discovery before routes are established. A Route Reply (RREP) is sent back to the source from the destination in order to establish a unicast route for data transmission.

The proactive OLSR [1] is one of the most popular protocols currently found in literature and experimental testbeds. It is a modified classical link state algorithm suited for requirements of MANET routing. OLSR introduces a flooding reduction technique using multipoint relays (MPRs) that are elected as being the minimum set of symmetrically connected 1-hop nodes that can symmetrically connect the source node to all 2-hop neighbours. Periodic HELLO messages are flooded 1hop to establish the MPR sets and periodic Topology Control (TC) messages are flooded network wide exclusively through MPR nodes to disseminate local route information globally. Consequently, this process helps towards populating routing entries for network wide destinations.

The Neighbourhood Discovery Protocol (NHDP), just recently RFC 6130, is a symmetric 1-hop and 2-hop neighbourhood discovery protocol for MANETs. This protocol requires each node to locally exchange HELLO messages so that each MANET node can detect the presence of bi-directional 1hop and 2-hop connected neighbours. This information is stored to determine direct connectivity to nodes while 2hop symmetric neighbourhood information is necessary for flooding optimisation techniques such as MPR selection in OLSR [1].

Dynamic MANET On-demand (DYMO) routing protocol [1] is the second generation version of AODV that is being developed at MANET WG. This protocol differs from AODV protocol in that it proposes using NHDP to detect bidirectional links during route establishment. DYMO also uses typelength-value (TLV) structure from the packet/message format described in RFC 5444 for generating routing packets. Relative to AODV, DYMO also support the Management Information Base (MIB), local route repairs, unicast links and accepts new improved routes even after routes establishment. OLSR version 2 (OLSRv2) is also a work in progress at the WG where OLSRv2 operates using the same basic algorithms and mechanisms as in OLSR but uses improved structures and control packet utilisation mechanisms. Particularly, OLSRv2 uses and extends NHDP for 2-hop node discovery and also uses the generalised packet/message format. The packet structural advancement includes the addition of MPR Address Block TLV containing the node MPR selection set and the corresponding willingness value.

\section{SCALABLE MANET ROUTING PROTOCOLS}

One of the main challenges towards deploying MANET networks is providing a scalable routing protocol that also guarantees the required QoS for packet delivery latency [2], [3], [4]. 


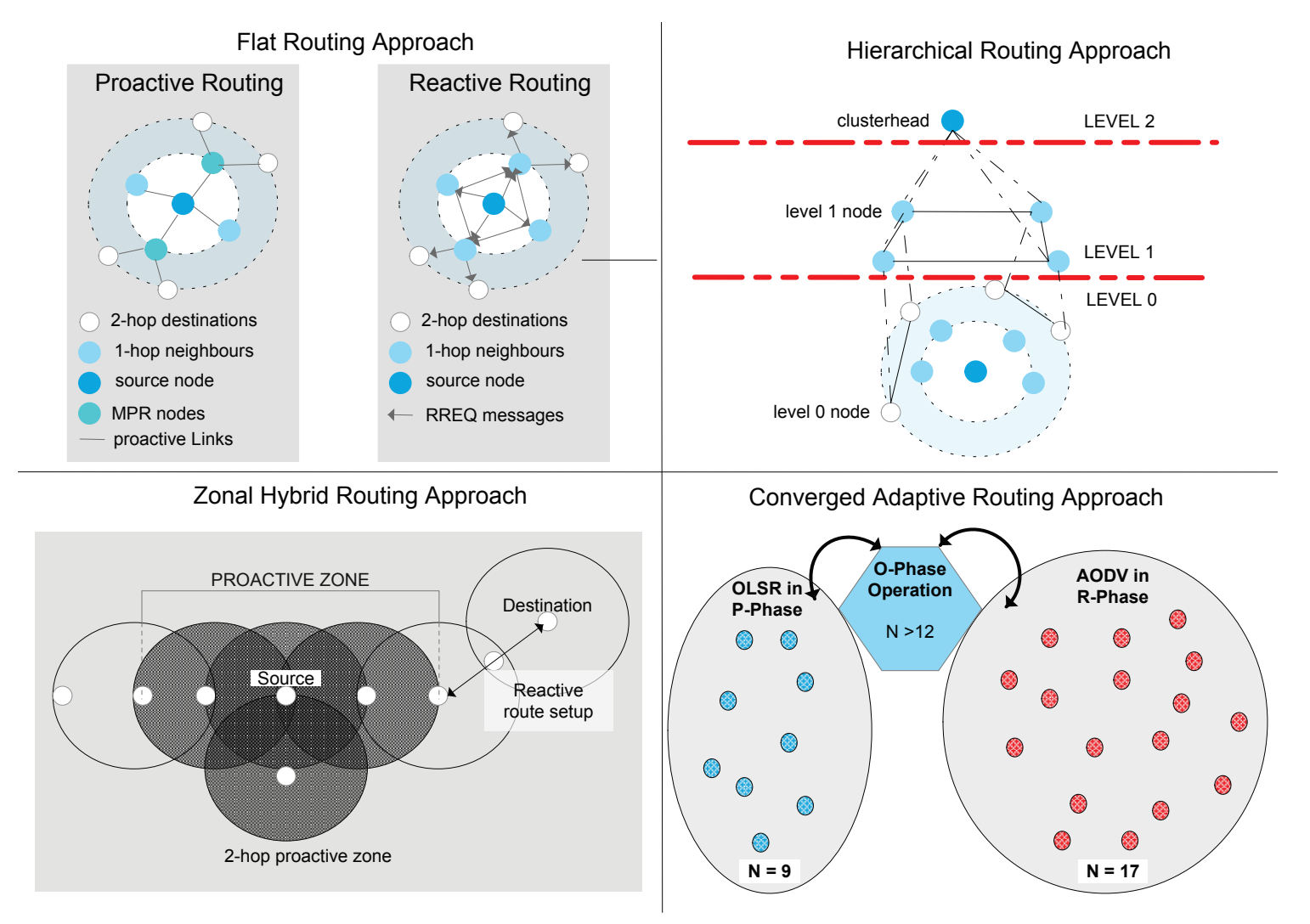

Fig. 2. Approaches for scalable MANET routing protocols

Routing scalability in the case of MANETs implies that the routing protocol needs to be able to sustain data routing with minimal delay and overhead for various increasing network sizes. There are several scalable MANET solutions proposed in literature as presented in [3], [4]. In this article, we present an integrated and updated overview of such solutions next, especially taking into consideration the recent advancements in the IETF MANET WG.

\section{A. Enhancements to Second Generation Routing Protocols}

OLSRv2 and DYMO are widely regarded as the second generation routing protocols for MANETs [1] as described above. The use of TLVs by the new generation protocols offers the possibility to efficiently add routing features so that the routing approaches are more scalable. OLSRv2 proposes the use of Fisheye State Routing features (FSR) through TLVs in order to exchange TC messages only with $k-h o p$ neighbours instead of flooding it over the entire network. The value of $k$ helps each given node to form a zone so that TC messages to faraway nodes are propagated with lower frequency than those of nearby destinations. This will result in OLSRv2 producing accurate paths information for $k-h o p$ neighbourhood of a node, and imprecise knowledge of paths to distant destinations. This imprecision is compensated by the packet route becoming more accurate as the packet approaches the destination. OLSRv2 also recommends Fuzzy Sighted Link State (FSLS) routing features additions for similar ends using the Hazy Sighted Link State (HSLS) for such optimisations. Thus, such approaches proposes to limit the TC message dissemination of OLSRv2 in space over time in order to achieve potential scalability.

\section{B. Nature Inspired Routing Approaches}

Moving away from traditional purely proactive and reactive approaches, there are several nature inspired algorithms that are being proposed as possible routing solutions to scalable MANETs such as ant colony optimisation, genetic zone routing and epidemic routing. One of the most well-known MANET nature inspired routing approach is the Adaptive nature-inspired algorithm for routing in mobile ad-Hoc Networks (AntHocNet). AntHocNet is designed based on selforganising behaviour of ant colonies with respect to shortest paths discovery as well as the related framework of ant colony optimization (ACO) [8]. The routing is achieved following ants approach of depositing a volatile chemical substance called pheromone moving between the nest and a food source, with higher pheromone intensity indicating shorter paths. These paths will then attract more ants and ultimately converge the majority of the ant agents onto a shortest path. This phase of global coordination of the agent actions is called stigmergy which is one of the key features of self-organised behaviours across various natural social systems including humans [8]. A good stigmergic model is believed to provide global robustness and scalability to the system, in our case, this should result in a robust and scalable distributed self-organized routing system.

AntHocNet is designed as a hybrid multipath algorithm consisting of both reactive and proactive components. It does not maintain paths to all destinations proactively, but sets up paths reactively where reactive forward ants (agents) are 
flooded by the source in order to find multiple paths to the destination, and backward ants (agents) return to establish these paths. The established paths are then represented in pheromone tables in terms of path quality. After reactive route establishment, data packets are routed stochastically over the different paths according to the path qualities. For a given data session, these routes are maintained and improved proactively using proactive forward ants (agents). Link failures are either repaired locally or by warning preceding route nodes of such an occurrence.

The Hierarchical OLSR model (HOLSR) [4] based on OLSR algorithm was proposed where node cluster levels are dynamically formed. This cluster structure supports random node mobility. HOLSR protocol thus reduces the amount of topology control information required at different hierarchical network topology levels, and the efficient use of existing high capacity nodes. In HOLSR, low-power nodes with only one interface are at Level 1, while nodes at the topology Level 2, are equipped with two interfaces, one of which communicates with Level 1 nodes. These longer range mobile nodes can also relay packets to other Level 2 simultaneously using different frequency band or medium-access control (MAC) protocol. The Level 3 nodes, are equipped with three wireless interfaces capable of communicating in turn with Level 1 , Level 2 and other Level 3 nodes at high-speed. There are clusters for each topology level, where MPRs are selected and TC messages exchanged. However, in HOLSR each interface sends out TC messages relating only to its own level and run HOLSR independently. Thus, hierarchal routing protocols tend to cluster mobile nodes to reduce area of flooding of topology messages using cluster head to manage routing in each virtual group of nodes.

\section{Hybrid and Adaptive Routing approach}

It has been demonstrated that considering the "best effort" routing protocols, OLSR is more suited to smaller networks as compared to AODV while AODV performs better for larger networks [5], [7]. The hybrid protocols combine both reactive and proactive features of traditional routing protocols such as OLSR and AODV. The proactive mechanisms are used to discover and maintain routes nearer to source nodes, reactive routing features are utilised for establishing routes with destination nodes located further away. Consequently, hybrid approaches tend to minimise the overhead introduced by proactive routing at the cost of added delay due to ondemand route discovery latencies before data transmission.

The Zone Routing Protocol (ZRP) [4] is among the first and most well-known hybrid MANET routing protocol. It is based on a routing zone concept where the minimum hop distance from a given node for proactive route maintenance is defined by the zone radius value, $k$. A value of $\mathrm{k}$ equal to 1 refers to 1-hop neighbourhood proactive route maintenance; while $\mathrm{k}$ is equal to the network diameter implies that the whole network will be covered proactively. ZRP was further enhanced into a zone routing framework using the independent zone routing (IZR) where each node configures its zone radius in a distributed manner using local measurements only in an adaptive manner.
Then, the Sharp Hybrid Adaptive Routing Protocol (SHARP) [9] claims to adapt efficiently and seamlessly between proactive and reactive routing strategies based on network characteristics. It can be configured to optimise user-defined performance metrics, such as loss rate, routing overhead, or delay jitter. A proactive zone is defined around some nodes with a node-specific zone radius determining the number of nodes within a proactive zone. All other destination nodes outside the proactive zone of a node is routed to using reactive routing approaches. Nodes found within a proactive zone maintain routes proactively only to a central node. It is important to note that SHARP creates proactive zones around popular destinations with data connections. Hence, SHARP reduces network wide overhead by focusing proactive routing overhead cost around popular destinations by dynamically adapting the zone radius of a node according to incoming data traffic and network mobility.

The novel ChaMeLeon (CML) [7] adaptive hybrid routing approach differs from the above described counterpart protocols in that it does not maintain routing zones. Instead, CML operates in three distinct phases of operation which are Oscillation $(\mathrm{O})$-phase, Proactive $(\mathrm{P})$-phase and Reactive (R)-phase. Each phase has augmented features that operate in parallel to traditional routing protocols. The CML design is focused towards providing a solution for hybrid adaptive routing protocols that are scalable whereby all the routing mechanisms is supported by an Adaptive Module. A threshold value for the size of the network is determined through experimentation. This is specific to deployment scenarios and has been found to be in the range of 10-15 nodes for the scenario defined in [7]. This threshold value indicates the network size point beyond which a reactive approach such as AODV is more efficient that the proactive OLSR protocol in terms of routing overhead, delay and delay jitter metric considerations.

Unlike ZRP and SHARP where zones delimit the proactive and reactive routing reaches for each node, CML dictates a converged network wide routing approach according to the size of the network in order to optimise routing overhead cost and improve QoS performance of the MANET routing process. Briefly, the default mode of operation is the P-phase whereby OLSR disseminates HELLO and TC messages. Each time such messages are received and the routing table updated, the adaptive module checks the number of reachable nodes in the network from the routing table, and compares it with the threshold value established. If the threshold is exceeded the $\mathrm{O}$ phase is initiated. The O-phase checks the oscillation timer and group oscillation limits before allowing or rejecting a phase shift initiation whereby the R-pahse is started with AODV routing and a CML alert message is flooded to converge the network to the required routing approach. The O-phase checks for the occurrence of oscillations i.e. if there are periodic group movement of nodes that causes network size to repetitively exceed the threshold. In such a case, repetitive phase shifts are deemed inappropriate. CML also defines a network size estimation algorithm utilised by the adaptive module in the $\mathrm{R}$ phase, such that if the network size shrinks back beyond the threshold, the O-phase can be re-instated. Thus, CML caters 
for dynamic scalable networks with temporal network size changes. A summary of the approaches is illustrated in Fig. 2.

\section{PERFORMANCE EVALUATION AND DISCUSSION}

In this section, we investigate the scalable performance of each routing approach with respect to a potential IoT deployment scenario and subsequently determine the relative suitability of each approach. We present simulation results using the network simulator 2 (ns2) [10] for our models corresponding to the routing overhead and data delivery delay from source to destination (measure of QoS for each approach as derived from [11]) as more devices join an adhoc collaborative network in real-time as will often be the case in the IoT. It is important to mention that a random waypoint mobility model was considered with average low mobility of $0.5 \mathrm{~m} / \mathrm{s}$ and high mobility of $1.5 \mathrm{~m} / \mathrm{s}$. Also, 10 discretely sourced UDP data connections over 802.11 MAC are used for these simulations while the default parameter values recommended from respective MANET WG RFCs and literature are implemented.

We present a simulation based delay and routing cost evaluation, in Fig. 3(a) and Fig. 3(c), to more analytically compare the described approaches as most have similar routing complexities but propose different optimisations to reduce cost. In Fig. 3(a), we illustrate a normalised measure of overhead for each approach for comparative ends. It can be seen that, for small network sizes of up to 10-12 nodes, all the approaches have similar scaling complexity of $O\left(N^{2}\right)$ and produce comparable routing costs. However, for larger networks each approach varies. Mainly, we have two trends emerging, one for proactive based protocols with optimisations (hybrid) and the second for reactive based trends. As it can be observed, OLSR has the highest cost because of its pure proactive approach scaling at $O\left(N^{2}\right)$. LAR has second worst cost because of the extra signalling required for geographical addressing, although some optimisation is achieved by targeted geographical flooding. ZRP uses predefined zones to limit proactive overhead, instead of geolocation, which further reduces cost compared to LAR. Then, AntHocNet uses mostly reactive routing to establish routes but has some elements of proactive route maintenance that add minimal costs as compared to ZRP. SHARP saves some costs as compared to ZRP given that it defines its proactive zones only around popular destinations. Interestingly, the optimisations provided by hierarchical routing paradigms based on OLSR has better performances that zonal hybrid approach. The segmentation of the proactive flooding domain significantly reduces the routing overhead and is only bettered by pure reactive DYMO routing in larger networks. Nevertheless, HOLSR type approaches requires node election in case of high mobility networks and can render it to be much more costly. As the network size grows the specified extra cost of proactive routing is further emphasised.

In Fig. 3(c), it can be observed that the same trend persists for higher mobility in the network and for the range of nodes of up to 100 nodes. In general, more routing overhead are generated by all approaches in order to keep updated routes providing the same level of QoS. Particularly, LAR and
HOLSR produces much more overhead coming from higher mobility network as a result of higher geographical and cluster based information maintenance respectively. Thus, CML tries to have an optimal reduction of routing overhead based on OLSR routing for smaller networks of less than 12 nodes and DYMO routing for larger networks. However, as observed in Fig. 3(a) and Fig. 3(c), there is a slight overhead that occurs during the switching period from P-phase to R-phase or viceversa due to network wide CML packet flooding to converge network routing.

Moreover, in Fig. 3(b), for larger networks (more than 12 nodes), it can be observed that OLSR, LAR and AntHocNet can only guarantee higher delay than reactive, hierarchical, zonal hybrid and hybrid adaptive approaches. However, proactive HOLSR has the delay performance comparable to DYMO with the drawback of having higher overhead especially for high mobility network as shown in Fig. 3(a) and Fig. 3(c). As the network size is increased, ZRP and SHARP reduces the delay caused by the proactive approaches by confining those to zones and using less delay prone reactive routing for interzonal communication. However, in smaller networks of less than 12 nodes, it is clear that since the same level of overhead is required by all approaches, the OLSR proactive route establishment causes decrease in overall end-to-end delay as compared to reactive and hybrid approaches that induces ondemand route establishment latency. The same trend occurs in higher mobility network, as results demonstrate in Fig. 3(d). Nonetheless, it can be observed that the overall delay increases as compared to results for low mobility in in Fig. 3(b). CML capitalises on this routing behaviour by using OLSR routing for networks smaller than 12 nodes and DYMO routing for larger networks. However, when CML detects 12nodes in the network and switches from P-phase to R-phase routing, a high data delivery delay is experienced at the time of the switching as shown in Fig. 3(b) and Fig. 3(d).

Thus, proactive routing approaches are vulnerable to higher delays in larger networks due to the high volume of packets it requires thus increasing medium access backoff wait times and queueing delays in addition to the multi-hop transmission delays. While for reactive approaches, the route discovery delay is reduced significantly because user diversity results in updated route information being available closer to the source. Therefore, a RREQ packet does not have to actually reach the destination to find the required route. Interestingly, for smaller networks, the proactivity of route establishments is more efficient than the DYMO on-demand based RREQ-RREP process. Thus, CML justifiably uses the OLSR approach for smaller networks while shifting to DYMO based routing for larger networks. However, as in the case of routing overhead, there is an increased delay experienced at the time the switch between phases occurs. This is due to the added delay at that particular point of convergence of network routing approaches.

\section{Conclusion And Future Work}

Scalability is one of the major hurdles towards deploying MANETs in the future IoT. MANET peripheral networks are useful in the sense that the distributed routing paradigm enabled by MANETs fits the requirement of self-managed and 


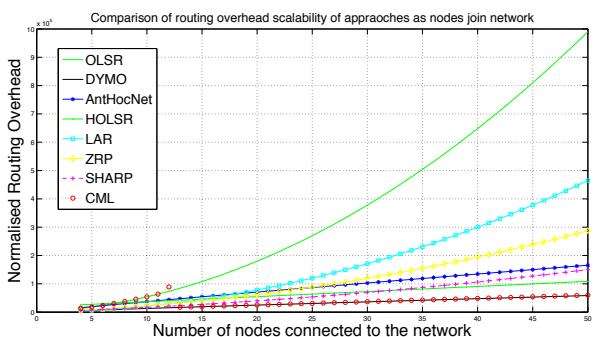

(a) Normalised routing overhead comparison as 30 nodes join the network with low mobility

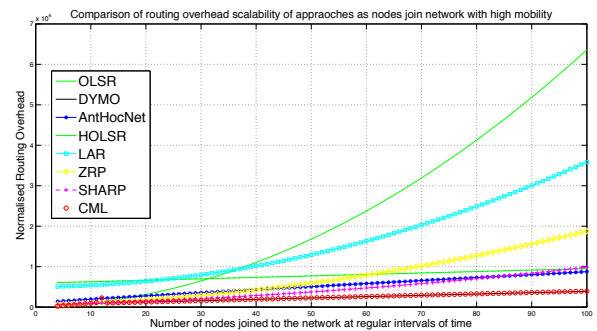

(c) Normalised routing overhead comparison as 100 nodes join (d) the network with high mobility

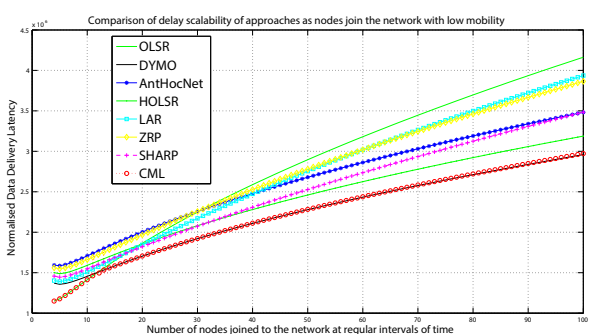

(b) Normalised routing delay comparison as 100 nodes join the network with low mobility

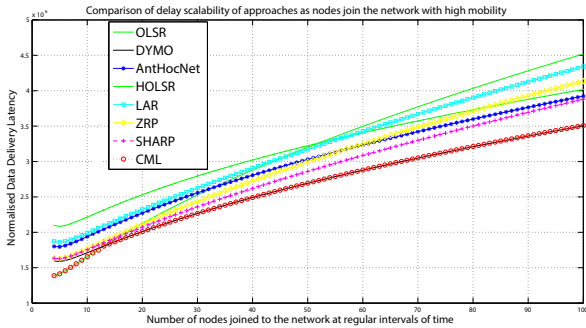

(d) Normalised routing delay comparison as 100 nodes join the network with high mobility

Fig. 3. Comparative Performance Evaluation of Scalability if OLSRv2, DYMO, AntHocNet, HOLSR, LAR, ZRP, SHARP and CML for nodes joining the network in low and high mobility scenarios

autonomic peripheral networks of small objects. Our investigative discussions in this article tends to indicate that a non-zonal adaptive approach should be further investigated where only one converged routing approach will be operating at a given time. The hurdle in such an approach appears to reside in the optimisation of the switching mechanism that should be rendered seamless, with low routing overhead cost and low impact on the routing QoS. Thus, future work that may help towards further reducing routing overhead and reduce data delivery latency include employing predictive algorithms for route lifetime determination and mobility based link errors.

\section{ACKNOWLEDGEMENT}

This work was supported in part by the European Unions FP7 project, ICT-619555 RESCUE (Links-on-the-fly Technology for Robust, Efficient and Smart Communication in Unpredictable Environments) and by the EU FP7 Project (Security and Interoperability for the Next Generation of PPDR Communication Systems) 2013 - 2016, Grant agreement no:313296

\section{REFERENCES}

[1] M. Conti and S. Giordano, "Multihop ad hoc networking: The theory," Communications Magazine, IEEE, vol. 45, no. 4, pp. 78 -86, april 2007

[2] S. Corson and J. Macker, "Mobile ad hoc networking (manet): routing protocol performance issues and evaluation considerations," Informational RFC 2501, January 1999.

[3] X. Hong, K. Xu, and M. Gerla, "Scalable routing protocols for mobile ad hoc networks," Network, IEEE, vol. 16, no. 4, pp. $11-21$, jul/aug 2002.

[4] A. Iwata, C.-C. Chiang, G. Pei, G. M., and T.-W. Chen, "Scalable routing strategies for ad hoc wireless networks," Selected Areas in Communications, IEEE Journal on, vol. 17, no. 8, pp. 1369-1379, August 1999.

[5] P. Samar, M. Pearlman, and Z. Haas, "Independent zone routing: an adaptive hybrid routing framework for ad hoc wireless networks," Networking, IEEE/ACM Transactions on, vol. 12, no. 4, pp. $595-608$, aug. 2004
[6] H. Cheng and J. Cao, "A design framework and taxonomy for hybrid routing protocols in mobile ad hoc networks," Communications Surveys \& Tutorials, IEEE, vol. 10, no. 3, pp. 62-73, march 2008.

[7] T. Ramrekha and C. Politis, "A hybrid adaptive routing protocol for extreme emergency ad hoc communication," in Computer Communications and Networks (ICCCN), 2010 Proceedings of 19th International Conference on, aug. 2010, pp. $1-6$.

[8] G. Di Caro, F. Ducatelle, and L. Gambardella, "Anthocnet: an adaptive nature-inspired algorithm for routing in mobile ad hoc networks," European Transactions on Telecommunications, vol. 16, no. 5, pp. 443$455,2005$.

[9] V. Ramasubramanian, Z. J. Haas, and E. G. Sirer, "Sharp: a hybrid adaptive routing protocol for mobile ad hoc networks," in Proceedings of the 4th ACM international symposium on Mobile ad hoc networking \& computing, ser. MobiHoc '03. New York, NY, USA: ACM, 2003, pp. 303-314. [Online]. Available: http://doi.acm.org/10.1145/778415.778450

[10] "The Network Simulator NS-2," http://www.isi.edu/nsnam/ns/.

[11] R. Groenevelt, P. Nain, and G. Koole, "The message delay in mobile ad hoc networks," Perform. Eval., vol. 62, pp. 210-228, October 2005. [Online]. Available: http://dx.doi.org/10.1016/j.peva.2005.07.018 